\title{
Microwave photonic frequency generation and conversion unit design for Ka-band satellite payloads
}

Georgios Charalambous, Stavros lezekiel

Georgios Charalambous, Stavros lezekiel, "Microwave photonic frequency generation and conversion unit design for Ka-band satellite payloads," Proc. SPIE 11852, International Conference on Space Optics — ICSO 2020, 1185243 (11 June 2021); doi: 10.1117/12.2599638

SPIE Event: International Conference on Space Optics - ICSO 2021, 2021, Online Only 


\section{International Conference on Space Optics-ICSO 2020}

Virtual Conference

30 March-2 April 2021

Edited by Bruno Cugny, Zoran Sodnik, and Nikos Karafolas
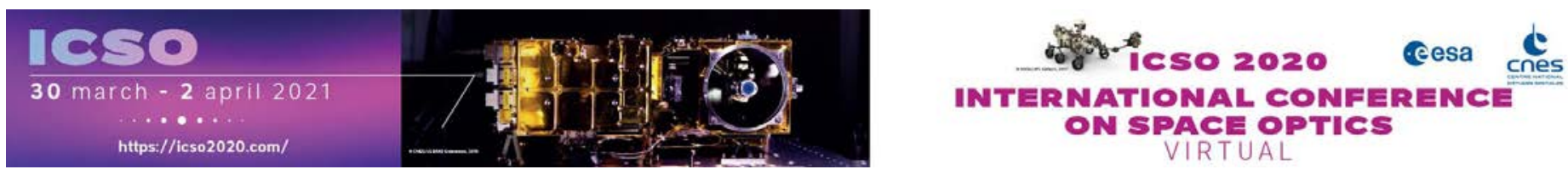

\section{Microwave photonic frequency generation and conversion unit design for Ka-band satellite payloads}

\section{Cesa isoporeasedngs denes}




\title{
Microwave Photonic Frequency Generation and Conversion Unit Design for Ka-band Satellite Payloads
}

\author{
Georgios Charalambous and Stavros Iezekiel \\ Microwave Photonics Research Laboratory, EMPHASIS Research Centre, University of \\ Cyprus, Nicosia, Cyprus \\ iezekiel@ucy.ac.cy
}

\begin{abstract}
A key challenge for HTS (high throughput satellite) providers is the provision of higher capacities whilst operating within a challenging mass-power-volume envelope. As payload requirements become more demanding, there is a move toward Ka-band, with even higher frequencies being used for Q/V-band feeder links. In order to satisfy the demand for reconfigurability and higher operating frequencies, there has been increased activity in microwave photonic (MWP) payload design. Two of the key functionalities that have been identified in microwave photonic payload design are the frequency generation unit (FGU) and frequency conversion unit (FCU). In this paper, we describe a proof-of-concept MWP FGU-FCU unit, which is configured as a breadboard demonstrator. The FGU is based on an X-band dual-loop optoelectronic oscillator, which provides a low phase noise microwave modulated LO to the following FCU stage. This unit is based on the application of electro-optic mixing in a Mach-Zehnder modulator. Incoming high frequency Ka-band signals (27.5-29.5 GHz) are applied to the RF drive of the EOM and are down-converted to a lower band (19.7-20.2 GHz) through subsequent photo-detection in the Optical-to-Electrical conversion $(\mathrm{O} / \mathrm{E})$ block. An optical amplifier $(\mathrm{OA})$ is placed in the photonic link to compensate the opto-electronic conversion losses at the optical domain of the income signals. Hence the EOM, $\mathrm{OA}$ and $\mathrm{O} / \mathrm{E}$ chain comprises the frequency conversion unit (FCU). We also outline potential paths to a fully integrated combined MWP FGU-FCU.
\end{abstract}

Keywords: high throughput satellite, optoelectronic oscillator, microwave photonic payload

\section{INTRODUCTION}

State-of-the-art satellite communication broadband networks are based on geostationary High Throughput Satellites (HTS). In the HTS architecture, a Gateway Earth Station (GES) services multiple spot beams employing frequency re-use and Circular Polarization orthogonality (RHCP and LHCP). Every spot beam employs a forward (FWD) channel/transponder that is used for the communication of the GES to the User Terminal and a return (RTN) channel/transponder for the communication of the User Terminal to the GES. This architecture results in a throughput increase by a factor of 20 or more compared to traditional satellite technology where a broad single beam is utilised to cover large regions/continents and the User Terminal and Gateway are required to be in the same beam. HTS are mainly used to provide broadband internet services to unserved or under-served regions with a bandwidth performance and cost comparable to terrestrial services.

Most HTS providers operate Ka-band fleets and to a lesser extent Ku-band. Ka-band HTS offer significant advantages over Ku-band counterparts, more specifically: Lower cost and higher capacity- this is due to the efficiencies resulting from the use of smaller sport beams which concentrate power and reuse spectrum in combination with Right Hand and Left Hand Circular Polarization (RHCP and LHCP). Comparing Ka-band HTS with $\mathrm{Ku}$-band broadcasting satellites, capacity increases by a factor of 20. Smaller and lighter flight equipment compared to lower frequency bands including passive and active devices (waveguides, filter banks, down-converters, amplifiers etc.) allows more capacity $(\mathrm{MHz})$ to be carried on a single spacecraft. This brings down the cost per bit and allows for more cost effective services.

State-of-the-art Ka-band HTS payloads offered by hardware manufacturers are typically implemented with transponders of $220 \mathrm{MHz}-480 \mathrm{MHz}$ bandwidth. In this work (the MIPHOSAT project), we consider a frequency plan along the following lines. In the forward (FWD) direction, i.e. communication from the Gateway to the User 
Terminal, a bandwidth of $2 \mathrm{GHz}$ (e.g. $27.5 \mathrm{GHz}-29.5 \mathrm{GHz}$ ) will be considered for the uplink part (i.e. GatewaySatellite) and $500 \mathrm{MHz}$ (e.g. $19.7 \mathrm{GHz}-20.2 \mathrm{GHz}$ ) for the downlink part (i.e. Satellite-User Terminal) employing in both cases $250 \mathrm{MHz}$ channels. Frequency reuse applies in the User Beam (UB) segment in combination with RHCP and LHCP. The same principle applies in the return (RTN) direction, i.e. communication from the User Terminal to the Gateway, where e.g. the band $29.5 \mathrm{GHz}-30 \mathrm{GHz}$ is used for the uplink path (User TerminalSatellite) and $17.7 \mathrm{GHz}-19.7 \mathrm{GHz}$ is used for the downlink path (Satellite-Gateway).

\section{MICROWAVE PHOTONICS FOR KA-BAND SATELLITE PAYLOADS}

Future satellite payloads will be required to offer re-configurability and higher bandwidths, with broadband communications forcing operators to anticipate the emergence of a new market provided that services are costeffective and flexible in the satellite industry for the next five to ten years. Photonic components are under consideration for such space-based platforms due to their many attractive attributes, such as low mass and large bandwidth. It is expected in the near-future that as microwave photonics matures, photonic devices and circuits will find increased penetration in satellite payload architectures, including frequency generation, translation, switching and signal processing. ${ }^{1-3}$ In this work, we focus on microwave photonics-enabled frequency generation and frequency conversion. The latter is based on electro-optic mixing in a Mach-Zehnder modulator (MZM) ${ }^{4}$ while the former is based on an optoelectronic oscillator (OEO). OEOs are an especially attractive proposition for FGUs since they offer very low noise performance that is not dependent on the oscillation frequency, with recent demonstrations up to the W-band. ${ }^{5}$

Typical payload architectures for the FWD channels are shown in Fig. 1. The signal transmitted in the FWD direction from the gate-way (GW) to the satellite payload is filtered and amplified with a low noise amplifier (LNA) before being fed to the down-converter $(\mathrm{D} / \mathrm{C})$. The $\mathrm{D} / \mathrm{C}$ is composed of a Ka-band mixer and a local oscillator converting the incoming high frequency Ka-band signals $(27.5 \mathrm{GHz}-29.5 \mathrm{GHz})$ to lower frequency $(19.7 \mathrm{GHz}-20.2 \mathrm{GHz})$ ones. Payload manufacturers usually design D/Cs composed of three sections: RF, MLO (master local oscillator) and Central Power Supply Unit. Down-converted signals are subsequently de-multiplexed to the user-beam (UB) frequencies before being amplified by high power amplifiers (HPA) based on TWTAs (travelling wave tube amplifiers). Signals are then filtered to remove any out-of-band nonlinear distortion and intermodulation products and are finally fed to the UB antennas for transmission. The return direction works in the reverse way.

The microwave photonic payload architecture for the FWD channel that is considered in the MIPHOSAT project is shown in Fig 2. The signal transmitted in the FWD direction from the GW to the satellite payload is filtered by a bandpass filter and amplified with an LNA before being converted to a modulated lightwave. An optoelectronic oscillator serves as the LO (i.e. it is the frequency generation unit, FGU), with its output being fed to the optical input of the electro-optic modulator (EOM) block, which comprises a Ka-band Mach-Zehnder modulator. Incoming high frequency Ka-band signals $(27.5 \mathrm{GHz}-29.5 \mathrm{GHz})$ are applied to the RF drive of the EOM and are down-converted to a lower band $(19.7 \mathrm{GHz}-20.2 \mathrm{GHz})$ through subsequent photo-detection in the Optical-to-Electrical conversion (O/E) block. Hence the EOM, Optical Amplifier (OA) and O/E chain comprises

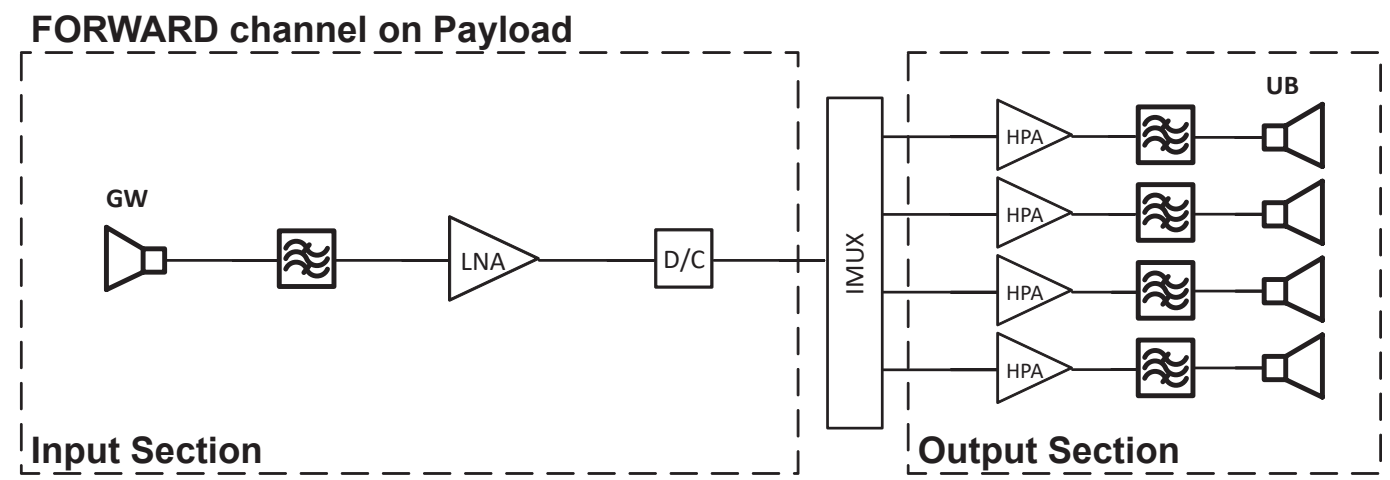

Figure 1. Forward channel architecture microwave payload. 


\section{FORWARD Channel on Payload}

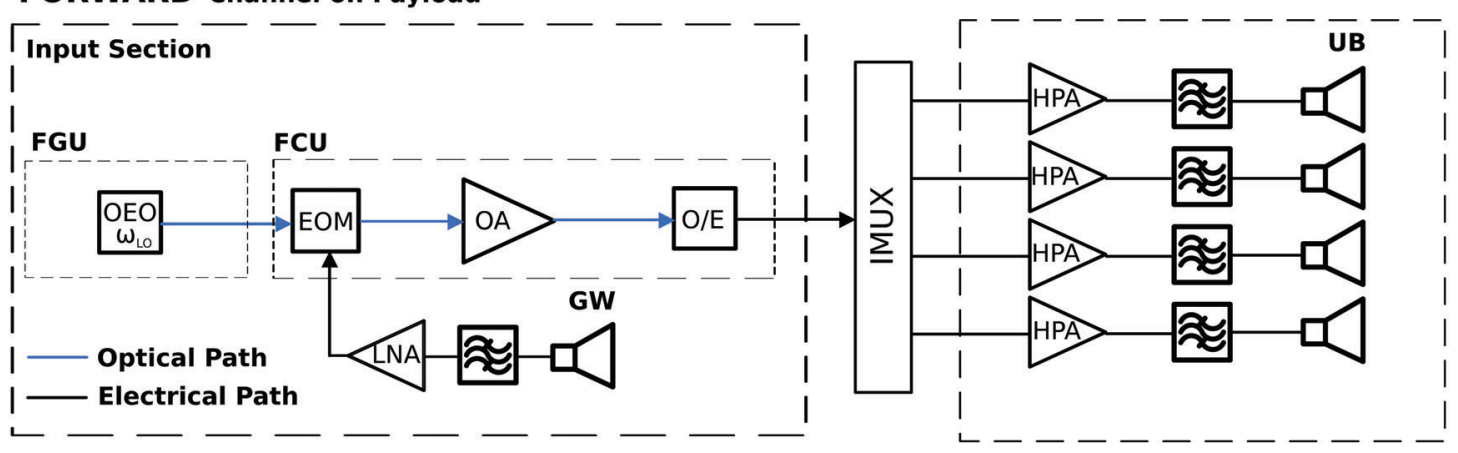

Figure 2. Forward channel architecture of a microwave photonic payload comprising an FGU and FCU.

the frequency conversion unit (FCU). In MIPHOSAT only the input section of Fig. 2 will be implemented (i.e. the FGU and FCU). However, in a full system, the down-converted signals are subsequently de-multiplexed to the UB frequencies before being amplified by HPAs. The out-of-band nonlinear distortion and intermodulation products are removed by filters before being fed to the UB antennas for transmission. The filters should not distort the in-band performance of the signal (e.g. gain and group delay).

\section{THEORETICAL STUDY}

In order to determine the conversion gain performance of the MWP-based FCU, a set of design equations and numerical simulations are essential in determining the impact of each individual FCU component. In the following sections, the models that are developed for the various FCU components can be used to maximise the conversion gain and to understand the output noise contribution on the fibre-optic-link (FOL). A down-converting FOL employing two quadrature-biased MZMs connected in cascade is shown in Figure 3. In this section, we evaluate the conversion loss of the FCU in which the optoelectronic oscillator (OEO) loop is open, and it is replaced by a frequency synthesizer to supply the LO signal. The X-band LO signal modulates the lightwave travelling through the MZM-1, creating two side-bands. This optical spectrum is fed through a second MZM-2, where it is modulated by the applied Ka-band RF signal. At the photodiode (PD), the signals are detected and the beating products generate the spur components and the intermediate frequency (IF).

The conversion loss (CL) of the mixer is defined as the power ratio between the IF and RF signals,

$$
C L=\frac{P_{I F}}{P_{R F}}
$$

while the input signal modulation index, expressed in radians for an RF signal is driven by:

$$
m_{R F}=\pi \frac{V_{R F}}{V_{\pi}}
$$

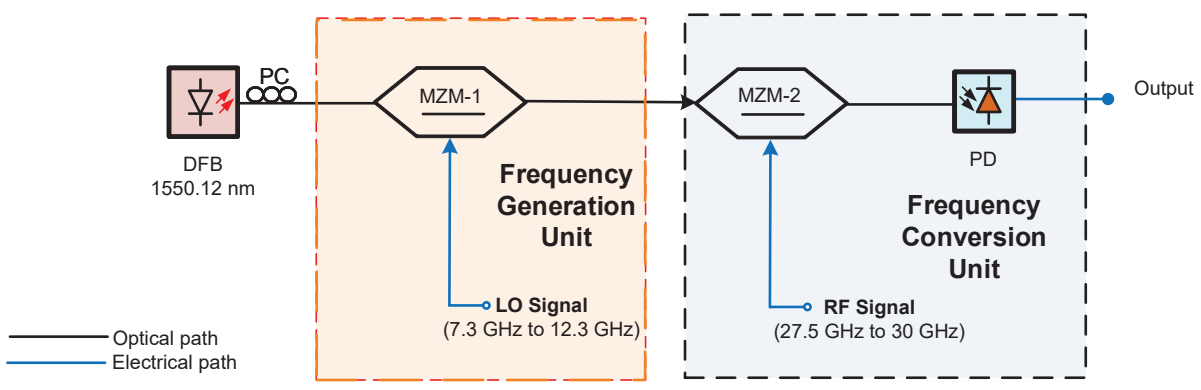

Figure 3. Simplified concept of the Frequency Generation Unit (FGU) and Frequency Conversion Unit (FCU) used for the mixer analysis. 
The microwave power of the received RF signal is usually unknown; the time-averaged input power at $\omega_{R F}$, referenced to an impedance $R_{i n}$, can be written as,

$$
P_{R F}=\frac{V_{R F}}{R_{\text {in }}}=\frac{V_{\pi}^{2} m_{R F}^{2}}{2 \pi^{2} R_{\text {in }}}
$$

where $V_{R F}$ and $m_{R F}$ are the amplitude and modulation index of the RF signal. After extensive calculations, the conversion loss is given by,

$$
P\left(\omega_{L O}-\omega_{R F}\right)=I_{p h}^{2}\left(2 J_{1}\left(m_{R F}\right) J_{1}\left(m_{L O}\right)\right)^{2} Z_{o}
$$

As shown in (4), the conversion loss is proportional to the square of the photodiode photocurrent, hence by increasing the photocurrent via optical amplification (taking care not to saturate the photodiode), the link conversion gain may be increased. (It is also possible to increase the photocurrent through microwave postamplification.) Tuning of the conversion gain is a function of the modulation index. However, in practice the modulation index of the incoming RF signal is unknown, therefore the modulation index of the LO is used as an optimisation variable to enhance $P\left(\omega_{L O}-\omega_{R F}\right)$.

As shown in Figure 3, various optoelectronic devices such as the laser diode, photodiode, and modulators comprise the FCU. Hence, the total output noise at the PD has contributions from the input noise, laser relative intensity noise (RIN), thermal, shot noise and the amplified spontaneous emission (ASE). Therefore, the link gains seen by the RF and LO signals are $g_{R F}=P_{I F} / P_{R F}$ and $g_{L O}=P_{I F} / P_{L O}$ respectively. The total output noise $\left(N_{\text {out }}\right)$ generated from the FCU is,

$$
N_{\text {out }}=\underbrace{k_{B} T B \cdot C L+k_{B} T B \cdot C L \cdot \frac{P_{R F}}{P_{L O}}}_{\text {Input Noise }}+4 k_{B} T B+2 q I_{p h} R_{L} B+R I N(f) \cdot I_{p h}^{2} R_{L} B
$$

where $k_{B}$ is the Boltzmann constant, $T$ is $290 \mathrm{~K}, B$ the RF bandwidth, $q$ the electron charge, and $R_{L}$ the load impedance. The noise-figure (NF) expressed at the downconverted signal is given by,

$$
N F=\frac{N_{\text {out }}}{C L \cdot k_{B} T B}
$$

and is strongly dependent on the CL of the FOL.

\section{EXPERIMENTAL SETUP AND RESULTS}

The self-oscillating mixing experimental set up is illustrated in Fig. 4. It consists of an OEO that provides an LO for the microwave photonic mixing section. The OEO is based on a dual loop topology using balanced photodetection. A continuous lightwave from the DFB laser is sent to the MZM-1 which is modulated by the feedback electrical signal. A bias controller is used to bias MZM-1 at quadrature, and compensates for drifts in bias. A 90:10 optical coupler is placed inside the loop in order to monitor the optical spectrum of the loop with an OSA. Then, a Y-branch coupler splits equally the lightwave signal from the output port of $90 \%$ optical coupler, routing the two outputs via optical fibres of $1 \mathrm{~m}$ and $100 \mathrm{~m}$ length, prior to photodetection in a balanced photodetector (BPD). At this point, the output microwave signal is multimode in nature; hence it is filtered by a microwave bandpass filter (BPF), and subsequently amplified by a cascade of three microwave amplifiers prior to a 10:90 microwave coupler. The $10 \%$ section is monitored using an ESA, while the remaining $90 \%$ of the signal power is fed back to the RF port of MZM-1.

The optical section of the OEO-based FGU is coupled to MZM-2 which is driven by a Ka-band RF synthesizer. The driving signal is up-converted to the optical domain and amplified using an optical amplifier (EDFA) to compensate for the losses from the optical sections (e.g. MZM-2 insertion loss) and for the opto-electronic conversion loss. Beating products between the oscillating signal and the Ka-band synthesiser are down-converted by the photodiode and measured with an electrical spectrum analyser (ESA) without additional microwave amplification and filtering. 


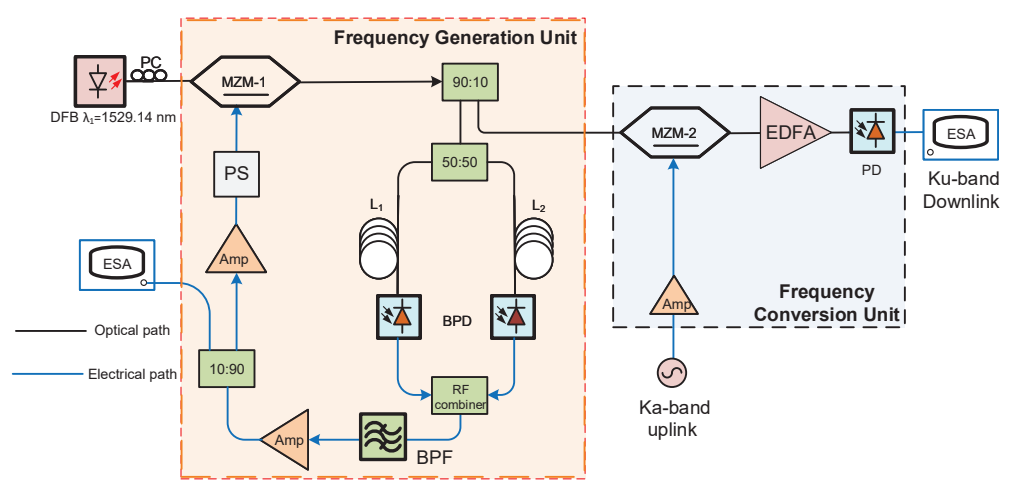

Figure 4. Experimental Setup of the Frequency Generation and Conversion Unit.

\subsection{Optoelectronic Oscillator}

The performance of the OEO-based FGU is determined from a set of experimental measurements in order to evaluate the impact of each individual component of the OEO. The laser power was set to $11 \mathrm{dBm}$ at $1550 \mathrm{~nm}$. The MZM is biased at quadrature $\left(V_{\pi}=4.9 \mathrm{~V}\right)$ while the path length difference was $100 \mathrm{~m}$. The OEO is optimised to sustain oscillations in the X-band. Fig. 5 shows the frequency spectrum of the OEO.
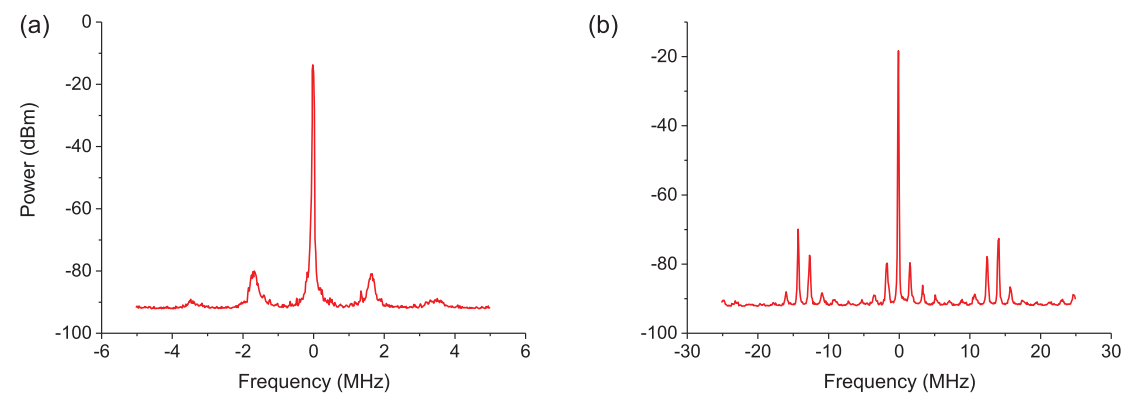

Figure 5. Optoelectronic Oscillator Spectrum at $10 \mathrm{GHz}$ for a SPAN of(a) $10 \mathrm{MHz}$ (b) $50 \mathrm{MHz}$

\subsection{Microwave Photonic Mixer}

\subsubsection{Photonic Mixer Conversion Loss}

Fig. 6 shows the detected IF power as a function of the input RF power for the three modulation indices $\left(m_{i}\right)$ of the MZM at the OEO section depicted in Fig. 4. As the modulation index $\left(m_{i}\right)$ increases, the conversion gain also increases as shown in Fig. 6.

For this proof-of-concept experimental setup, we used an RF of $22 \mathrm{GHz}$ (dictated by the highest operating frequency of the synthesizer) and a power level of $0 \mathrm{dBm}$. A frequency doubler was used subsequently in order to extend the frequency range of the RF signal in order to meet the original requirement of an RF signal up to $30 \mathrm{GHz}$. Frequency multiplication, however, leads to SNR degradation and hence this approach was not used initially. The OEO-based FGU provides an LO at approximately $10 \mathrm{GHz}$ with the spectrum as shown previously, in Fig. 5. The spectrum of the downconverted signal (corresponding to an IF at $12 \mathrm{GHz}$ ) and the two spurious frequency products, namely the RF (incoming signal) and LO (OEO), are shown in Fig 7(a). Since the frequency spacing between the IF, RF and LO signals is large, the spurious signals may be suppressed with a microwave bandpass filter at the IF signal.

The spurious signals (from the RF synthesizer and LO/OEO) are shown in Fig 7(b-c). Inspecting the downconverted signal at $12 \mathrm{GHz}$ (Fig 7(d)), the peak power of the IF signal is $-63 \mathrm{~dB}$ relative to the $0 \mathrm{dBm}$ input signal from the RF frequency synthesiser, giving a conversion loss (CL) of $63 \mathrm{~dB}$. This is based on the definition in equation (1). 


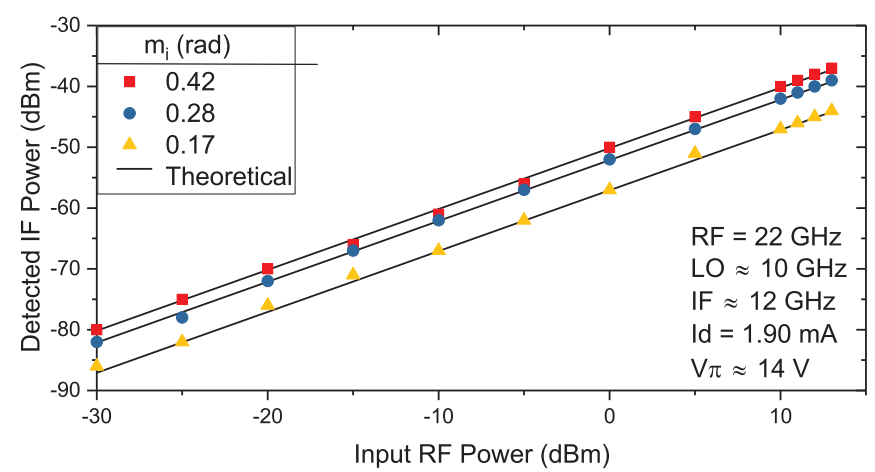

Figure 6. Conversion loss performance of the down-converting link for a total detector current of $1.9 \mathrm{~mA}$ at an RF frequency of $22 \mathrm{GHz}$. Circles are measured data points, solid lines are modelled. A $50 \Omega$ load resistance was assumed in the model (4).
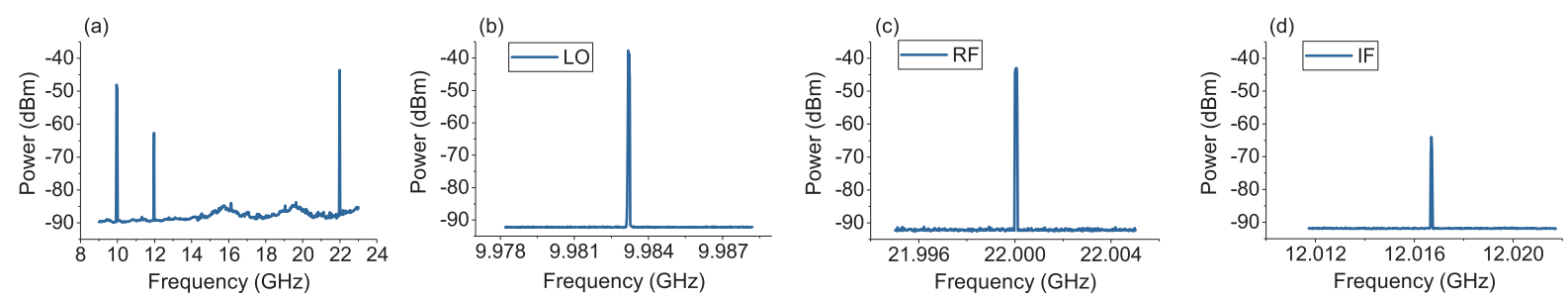

Figure 7. (a) Down-converted signals at the output section of the MWP mixer. Spurious signals at the output section of MWP mixer(b)RF and (c) LO. (c) Downconverted IF signal (22 GHz - $10 \mathrm{GHz})$.

\subsubsection{Output Noise at the IF}

The output noise level at the photodiode is depicted in Fig. 8, where the shot noise and thermal noise limited regimes are clearly identified, as is the impact of varying the laser RIN. For the experimental setup of Fig. 4, the output power level of the laser was held constant (RIN is bias dependent) and the photocurrent was increased (by increasing the optical gain of the EDFA). The bandwidth at the photodiode section is normalized to $1 \mathrm{~Hz}$ while the CL, LO and RF power are $50 \mathrm{~dB}, 10 \mathrm{~mW}$ and $1 \mathrm{~mW}$ respectively. The measured data points indicate that the photodiode is RIN-limited since they follow the trend line of $-135 \mathrm{~dB} / \mathrm{Hz}$ for RIN. The laser RIN is poor $(-135 \mathrm{~dB} / \mathrm{Hz}$ from the manufacturer's data sheet), thus the output noise levels may be decreased by using a better laser source.

Fig. 9 shows the measured detected IF power while the input RF power at the MZM microwave electrode was varied. The solid lines correspond to the conversion loss model from (4), with the parameters shown in the inset. From Fig. 10, we can calculate the minimum detectable signal (MDS) and noise figure (NF). The slope of the blue line gives the conversion loss without microwave gain, while the red line shows the conversion loss with an additional microwave gain of $26 \mathrm{~dB}$ (with a microwave amplifier subsequently connected to the photodiode). The $\mathrm{RF}$ and $\mathrm{LO}$ frequencies are $22 \mathrm{GHz}$ and approximately $10 \mathrm{GHz}$ respectively, for a $2 \mathrm{~mA}$ total PD current, and the $\mathrm{NF}$ and MDS of the down-converting MWP mixer are calculated to be $86 \mathrm{~dB}$ and $-86 \mathrm{dBm}$, respectively. For a microwave gain of $36 \mathrm{dBm}$, as shown in Fig. 10, the calculated NF and MDS improve to $56 \mathrm{~dB}$ and $-123 \mathrm{dBm}$ respectively, due to the improvement in conversion gain.

A second signal generator was used to produce another RF signal in order to measure the spurious free dynamic range (SFDR). An LO (OEO) power of $0 \mathrm{dBm}$ at $10 \mathrm{GHz}$ was chosen, and the RF two-tone signals were set to $2.9 \mathrm{GHz}$ and $3 \mathrm{GHz}$ (corresponding to a frequency spacing of $100 \mathrm{MHz}$ ). Varying the two-tone power levels, the output power of the downconverted fundamental terms and the third-order intermodulation distortion (IMD3) were measured. These are plotted in Fig. 10 (blue and red dots). The data were fitted linearly $(y=a x+b, \quad$ where $a=2.93$ and $b=-106)$. The slope $(a=3)$ of the fitted model verifies that we tracked IMD3. As shown in Fig. 10, the measured noise floor is $-141 \mathrm{dBm} \mathrm{Hz}^{-1}$ and the SFDR is $80 \mathrm{~dB} / \mathrm{Hz}^{2 / 3}$ (without a microwave filter). 


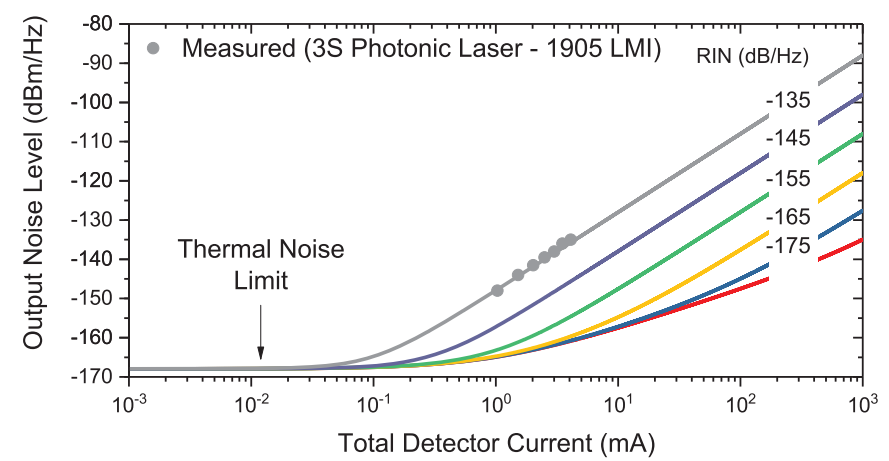

Figure 8. Mathematical model from (5) and measured microwave photonic mixer IF output noise levels as a function of total detector current for different levels of laser RIN.

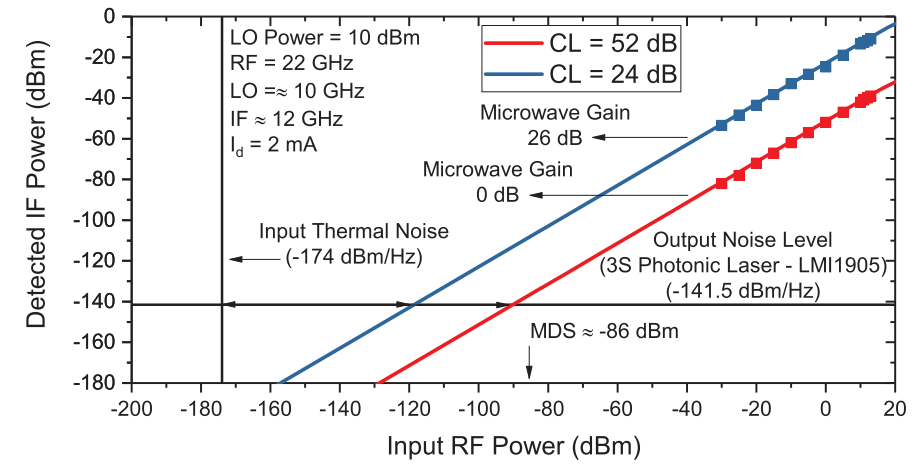

Figure 9. Performance of the down-converting link for a total detector current of $2 \mathrm{~mA}$. The NF and MDS are for a carrier frequency of $22 \mathrm{GHz}$.

\section{CONCLUSIONS}

For the experimental OEO set up, we measured a fixed centre frequency of $10 \mathrm{GHz}$, a side-mode suppression of $70 \mathrm{~dB}$, and an SNR of $80 \mathrm{~dB}$, with $2 \mathrm{MHz}$ of FSR and output power of $0 \mathrm{dBm}$. A proof-of-concept microwave photonic mixer was implemented and used to down-convert a Ka-band signal at $30 \mathrm{GHz}$ to an IF at $20 \mathrm{GHz}$, using a $10 \mathrm{GHz}$ OEO. A maximum conversion gain of $-50 \mathrm{~dB}$ was measured with a corresponding photocurrent of only $2 \mathrm{~mA}$. The measured carrier-to-noise ratio of the IF signal is $45 \mathrm{~dB}$, and the measured SFDR is $80 \mathrm{~dB} / \mathrm{Hz}^{2 / 3}$ (without a microwave filter). Placing a bandpass filter at the IF leads to a further $40 \mathrm{~dB}$ suppression of the IMD products, leading to a final value of $100 \mathrm{~dB} / \mathrm{Hz}^{2 / 3}$. A NF of $85 \mathrm{~dB}$ and $56 \mathrm{~dB}$ is calculated for a conversion loss of $52 \mathrm{~dB}$ and $24 \mathrm{~dB}$, respectively. Increasing the photocurrent or the microwave gain, the conversion loss is reduced, leading to an improved NF.

\section{ACKNOWLEDGMENTS}

We thank F. Deborgies (European Space Agency) for his continuous support and guidance. The work reported here was conducted as part of ESA Contract 4000124371/18/NL/SC. The view expressed herein can in no way be taken to reflect the official opinion of the European Space Agency.

\section{REFERENCES}

[1] Sotom, M., Aveline, M., Barbaste, R., Benazet, B., Kernec, A. L., Magnaval, J., and Picq, M., "Flexible photonic payload for broadband telecom satellites: from concepts to system demonstrators," in [International Conference on Space Optics - ICSO 2016], Cugny, B., Karafolas, N., and Sodnik, Z., eds., 10562, 558 566, International Society for Optics and Photonics, SPIE (2017). 


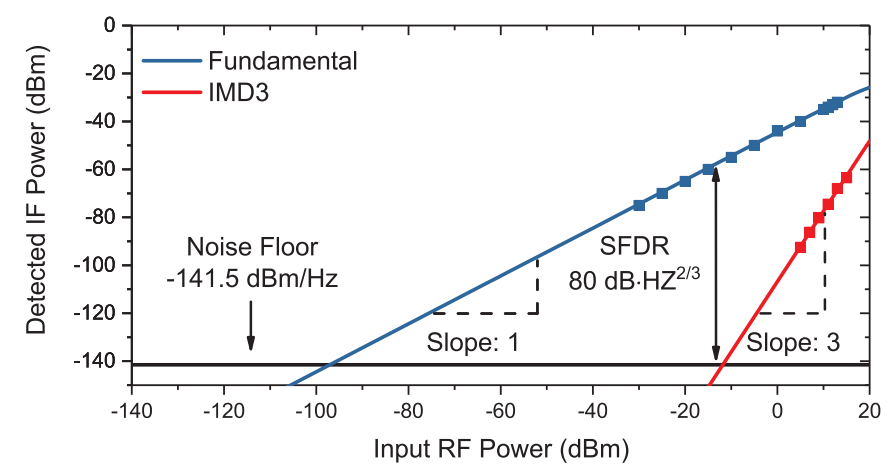

Figure 10. Spurious free dynamic range for the fundamental frequency (IF) and IMD3 as a function of the power level of the two-tone RF input.

[2] Anzalchi, J., Inigo, P., and Roy, B., "Application of photonics in next generation telecommunication satellites payloads," in [International Conference on Space Optics - ICSO 2014], Sodnik, Z., Cugny, B., and Karafolas, N., eds., 10563, 1063 - 1071, International Society for Optics and Photonics, SPIE (2017).

[3] Sotom, M., Benazet, B., Le Kernec, A., and Maignan, M., "Microwave photonic technologies for flexible satellite telecom payloads," in [2009 35th European Conference on Optical Communication], 1-4 (2009).

[4] Pan, S., Zhu, D., Liu, S., Xu, K., Dai, Y., Wang, T., Liu, J., Zhu, N., Xue, Y., and Liu, N., "Satellite payloads pay off," IEEE Microwave Magazine 16(8), 61-73 (2015).

[5] Hasanuzzaman, G. K. M., Iezekiel, S., and Kanno, A., "W-band optoelectronic oscillator," IEEE Photonics Technology Letters 32(13), 771-774 (2020). 